\title{
Research and design of interface in marine navigation information system
}

\author{
Jun Wan \& Zhiming LV \\ School of Mechanical and Electrical Engineering, Chongqing Creation Vocational College, China
}

Keywords: Marine navigation; Information system; Interface.

\begin{abstract}
Marine navigation information system is an indispensable modern shipping navigation equipment. With the continuous development of the international shipping industry, new demands have come up in real-time, stability and versatility, etc., which puts forward the higher request to the ship navigation system. In recent years, however, the relevant research focused on how to use the latest GIS positioning and communication technology to realize more precise, real-time navigation function. However the research on how to design and implement such navigation system is less. This article focus on the current new development of ship navigation sys-tem, studies the actual demand of the system, analyzes the structure and the types of the information system to deal with, and some key interfaces for navigation information system is studied and designed. On this basis, the preliminary implementation and simulation are carried on, proving the feasibility of the proposed scheme, also proving the certain basis for the future navigation information system design and manufacturing.
\end{abstract}

\section{Introduction}

Ship navigation information system integrated with computer, communications, signal processing, automatic control and other fields of expertise, is indispensable in modern shipping navigation equipment, since the advent of the last century, with the advantages of accuracy, real-time, high efficiency, rapid application and popularize[1], and therefore, in the field of academic and engineering fields for Marine navigation technology and the research achievements of navigation system is relatively more, gradually developed into a relatively mature technology. In recent years, due to the rapid development of information technology, on the one hand, brought new life for ship navigation technology, on the other hand also puts forward a new challenge for ship navigation information system. With a variety of new technology was introduced into the system, greatly increase the complexity of the system, at the same time due to the continuous development of shipping industry and user needs changing, make original varieties single function of ship navigation system gradually evolution in the direction of differentiation, more functional. Under this development trend, on how to design and implement complex and varied functions, stable and reliable performance of ship navigation information system, research is lacking, this paper is focus on the system of the practical need of and implementation, the related problems are studied and discussed. In the first section of this article, mainly introduces the background of ship navigation information system, as well as the realization of key point; In the second section, the study of navigation system to deal with the sort of information; In the third section, the study of information system in the interface and the actual; In the fourth quarter, based on the proposed scheme, the software realization of the preliminary Finally, gives the summary of this article and the next step.

\section{Ship navigation information system}

For different types and USES of the ship, for navigation information systems have different requirements, such as for large ships, the information such as the latitude and longitude, speed, heading for the ship have a more accurate demand, and real time is not too high requirements for information; For small vessels, it is often found in the busy port, need real-time navigation information, and avoid other ships, avoid collisions, and thus have high requirements for navigation update rate; And for special purpose ships, often need to deal with the bad sea conditions, insist on 
ship navigation information system has good stability and reliability. Because of this, so far no internationally accepted design standards of ship navigation information system.

In the system, need to use hardware, such as GPS chip, realize the corresponding function, the current hardware equipment technology is mature, the corresponding interface has been standardized, application is more convenient [3]. However, how to deal with all sorts of equipment information collection, and its synthesis is made valid by means of the navigation information, is the difficulty of the ship navigation system implementation, therefore, the core of the system software design. And the design of the software interface is the key to connect system parts, as shown in the figure below, in this paper, system interface design can be divided into three levels.

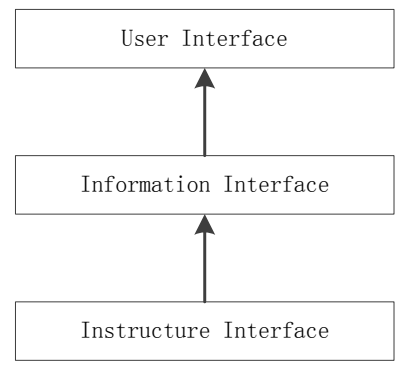

Fig.1 Design of the system interface

Applications such as introduced above, the current need of hardware, such as GPS systems, such as ship velocimeter, its interface has been development mature, use more convenient, do not need to carry on the secondary development. And user interface design, due to different user demand, in the process of actual implementation, need according to the specific requirements in terms of development. Interface is the key point of this article focuses on the information, on the one hand, through the interface to the hardware the collected information to navigation information system of the central processing module, on the other hand the upper user interface by calling the corresponding information interface, information transmission, display, analysis, and specific functions. Therefore, information interface is the core of the navigation information system, the paper will mainly study and design of this kind of interface.

\section{Navigation information analysis}

Handling and storage of a variety of Marine navigation information system information, first of all, the system monitoring information, submitted to deal with hardware interface, and gives the corresponding hardware equipment according to the requirements of the upper user feedback; Second, the system will need to process and synthesis of information display to the user, at the same time, from the user input device to collect the user's needs and processing; Finally, the system also needs and GIS, satellite communication system, such as interaction module, the analysis of the information and update[4]. Navigation information, therefore, in the process of the flow of the system, formed the three closed loop, as shown in the figure below:

In hardware information closed loop, the hardware interface of information mainly for the latitude and longitude, heading, speed, such as initial information, and through the hardware interface and output the information itself. However, due to the different standards and different devices used by different manufacturers, and its data format differences, information interface cannot be used directly, so need information collection and formatting module for data format. The module to collect data from the hardware interface, and according to the pre-defined data structure is data organization, and submit to the information receiving interface in a certain format, in the interface of receiving information, is to test the validity of the data, and identify the type of data, delivered to the corresponding central processing function. Information interface design

In the second section, information on the application of the system interface are introduced, and its function are discussed in detail, according to its function and position is different, it can be divided into three categories: the information receiving interface, interface of information delivery, 
information extension interface. In this section, the research and design of the three kinds of interface in detail.

\subsection{Information receiving interface}

Information receiving interface's main function is: to receive the information submitted by the hardware, and test the format of the information and classification, finally submitted to the central processing module. Its main structure and working process as shown in the figure below:

as shown in the interface of receiving information, information stored in the message buffer first, received information format pattern matching unit through a predefined legitimate message format, to match the information in the message buffer, monitoring its legitimacy, when the abnormal feedback control unit scheduling message buffer, according to the source of the exception message to the corresponding hardware interface for feedback, which will be discarded the exception message or closed/reset the corresponding hardware interface. Legitimate messages are fed into the classification unit, the unit to maintain several list, the list of message data storage format. When the central processing module or other modules, call the corresponding function, through the function of the corresponding interface, remove the corresponding data and give the corresponding function.

\subsection{Information delivery interface}

The is the underlying hardware interface for receiving information equipment and systems, and information delivery interface for the upper user and user interface, its features are: the central processing module of processed data and information, according to the different types of presented to the user interface, to integrate and display in the user interface. Its main structure and working process as shown in the figure below:

And the difference of the interface receiving information, delivery interface for the two-way information transmission function. First, unit calling through the data processing can be completed data delivered to the user interface is used, depending on the type of a graphical data, byte data, GIS data and so on, the user interface by calling these different kinds of data, can according to the actual demand, the synthesis and showed that contains rich content of the navigation information here.

Information delivery interface is the foundation of the realization of the user interface, the don't care about is how to implement the user interface, the end user has what kind of specific needs, merely provides a series of standardized data delivery interface, so you can according to different types of vessels and different application scenarios, different user interface design, and don't need to be under the constraints of the core software system.

\subsection{Information extension interface}

Extension and the former two kinds of different interface, information interface is open to the outside interface, through the interface, such as satellite communication network, GIS system, AIS system, etc., all can be connected into the navigation information system, for the system to provide more abundant information and more powerful. Its main structure and working process as shown in the figure below:

Information extension interface also need to take the bidirectional data transmission task, on the one hand, its operation has a corresponding data exchange/transport protocol stack, such as AIS data transmission protocol stack, the VSAT satellite communications protocol stack, etc., in order to download interface and transmitting messages through the corresponding system, at the same time converts received message system can identify the data structure, for the central processing module. System, on the other hand, according to the need, put forward the corresponding information to download the request, the request through the request processing unit, is converted to the corresponding system control and request instructions, encapsulated in the appropriate control message, sent to the corresponding external systems.

Information extension interface can be extended external data exchange protocol stack to extend its can support the types of external system, comprehensive more external information, so that they can enrich the content of navigation information system.

\subsection{The simulation and realization}

According to the research and design of any of the above section, this article adopts the method of software realization on the navigation information system are simulated and the simulation. USES the 
environment is the Core i3 $1.8 \mathrm{Ghz}, 4$ gb of memory, Windows 764 - bit, using Java as a programming language.

Simulation of the key is to realize the function of each interface, verify the effectiveness of each interface work and efficiency, based on the current relatively mature central processing module, implements for the processing and storage of information, and the whole simulation system is developed using Java language, simulation of the system interface as shown in the figure below:

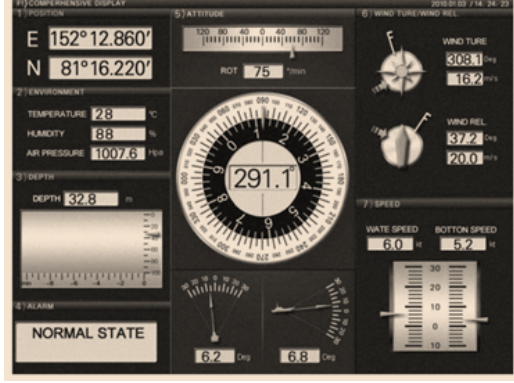

Fig.2 The interface of the system

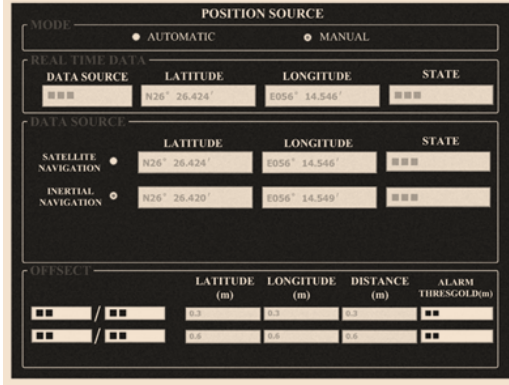

Fig.3 The frame of system configuration

By writing the corresponding code, simulate all kinds of hardware, interface successfully receiving information collection and conversion of different kinds of news, and information delivery interface presented to the user interface, can be seen from the above, it successfully realized the information collection and display.As shown in the figure below, the information set test, through the design of the control panel, set the corresponding parameters, can observe the system for effective control.

Through the implementation of the above prove that, in theory, in this paper, the design of the interface can display the corresponding function, realize the navigation information collection, processing and delivery functions, illustrates the feasibility of the proposed scheme has some.

\section{Conclusion}

This article in view of the current new development of ship navigation system, studies the actual demand of the system, analyzes the structure and the types of the information system to deal with, and some key interface for navigation information system is studied and designed, and on this basis, carried on the preliminary implementation and simulation, proved the feasibility of the proposed scheme, at the same time for the future navigation information system design and manufacturing have provided the certain basis.

In the next step of work, through practical scheme proposed in this paper, in practice to verify the actual function of each interface, and the design method of each interface for further optimization, eventually to build a fully functional, stable performance of ship navigation information system.

\section{References}

[1] Ben Shneiderman, Catherine Plaisant, Maxine Cohen, Steven Jacobs. Designing the User Interface: Strategies for Effective Human-Computer Interaction (3th Edition) (M). USA: Publishing House of Electronics Industry, 2004.66-78.

[2] Fang quangen. Bridge Resource Management (I" Edition (M)). Beijing: China Communication Press, 2006. 32-44.

[3] Wu jianhua, Modem information system of navigation (I" Edition) (M).Wuhan: Wuhan University of Technology Press, 2007.22-23.

[4] Zhu zuxiang, Ge liezong, Zhang zhijun. Engineering psychology (l"Edition) (M), Beijing: People education press, 2000, pp.209-215. 